Review began 07/17/2021 Review ended 08/23/2021 Published 08/23/2021

๑) Copyright 2021

Gubod et al. This is an open access article distributed under the terms of the Creative Commons Attribution License CC-BY 4.0., which permits unrestricted use, distribution, and reproduction in any medium, provided the original author and source are credited.

\section{Oral Lichen Planus in a Patient With a Thymoma: A Rare Finding}

EB Robson Gubod ${ }^{1}$, Anand Ramanathan ${ }^{2,1}$, Zuraiza Mohamad Zaini ${ }^{1}$, Saman Warnakulasuriya ${ }^{3}$

1. Oral \& Maxillofacial Clinical Sciences, Faculty of Dentistry, University of Malaya, Kuala Lumpur, MYS 2. Oral Cancer Research \& Coordinating Centre, Faculty of Dentistry, University of Malaya, Kuala Lumpur, MYS 3. Faculty of Dentistry, Oral \& Craniofacial Sciences, King's College London, WHO Collaborating Centre for Oral Cancer, London, GBR

Corresponding author: Anand Ramanathan, drranand@um.edu.my

\section{Abstract}

We present a rare case of ulcerative oral lichen planus that was associated with a thymoma discovered during the management phase, seven months after the initial diagnosis of oral lichen planus. Thymectomy was performed and investigations revealed pure red cell aplasia. Although rare, the association of a thymoma should be considered in recording the medical history of patients presenting with oral lichen planus.

Categories: Allergy/Immunology, Oncology, Other

Keywords: oral lichen planus, thymoma, pure red cell aplasia, topical steroids, cyclosporin, management

\section{Introduction}

Oral lichen planus (OLP) is a chronic inflammatory disease of the oral mucosa which affects 1 - $2 \%$ of the population [1]. Thymoma is a neoplasm of the thymus epithelial cells, which is commonly associated with autoimmune diseases such as myasthenia gravis and systemic lupus erythematosus [2]. OLP associated with thymoma is rarely seen in clinical practice. Here we report a case of lichen planus associated with thymoma which was discovered seven months after the diagnosis of OLP.

\section{Case Presentation}

A 45-year-old Chinese man presented with the complaint of pain and burning sensation in the mouth associated with hot and spicy food consumption for an approximately one-month duration. At presentation, he had no known systemic illness and was not on any medications. He occasionally consumed alcohol and had never smoked. There was also no history of usage of a new toothpaste, new mouthwash, and/or undergoing dental restoration work before symptoms appeared. At the initial visit, there were no skin, genital, scalp lesions, or nail abnormalities. He did not have any ptosis.

On examination, the patient presented with symmetrical, white interlacing striations with erythema and superficial ulcerations on bilateral buccal mucosal surfaces. Erythema and ulceration were noted on the left lateral border of the tongue along with keratotic plaques on the dorsal surface of the tongue (Figure 1). No induration was noted. Other mucosae were unaffected. Several dental restorations composed of glass ionomer cement and composite resin were noted adjacent to the lichenoid lesions on the buccal mucosa. A provisional diagnosis of OLP/ oral lichenoid reaction was established. 


\section{Cureus}

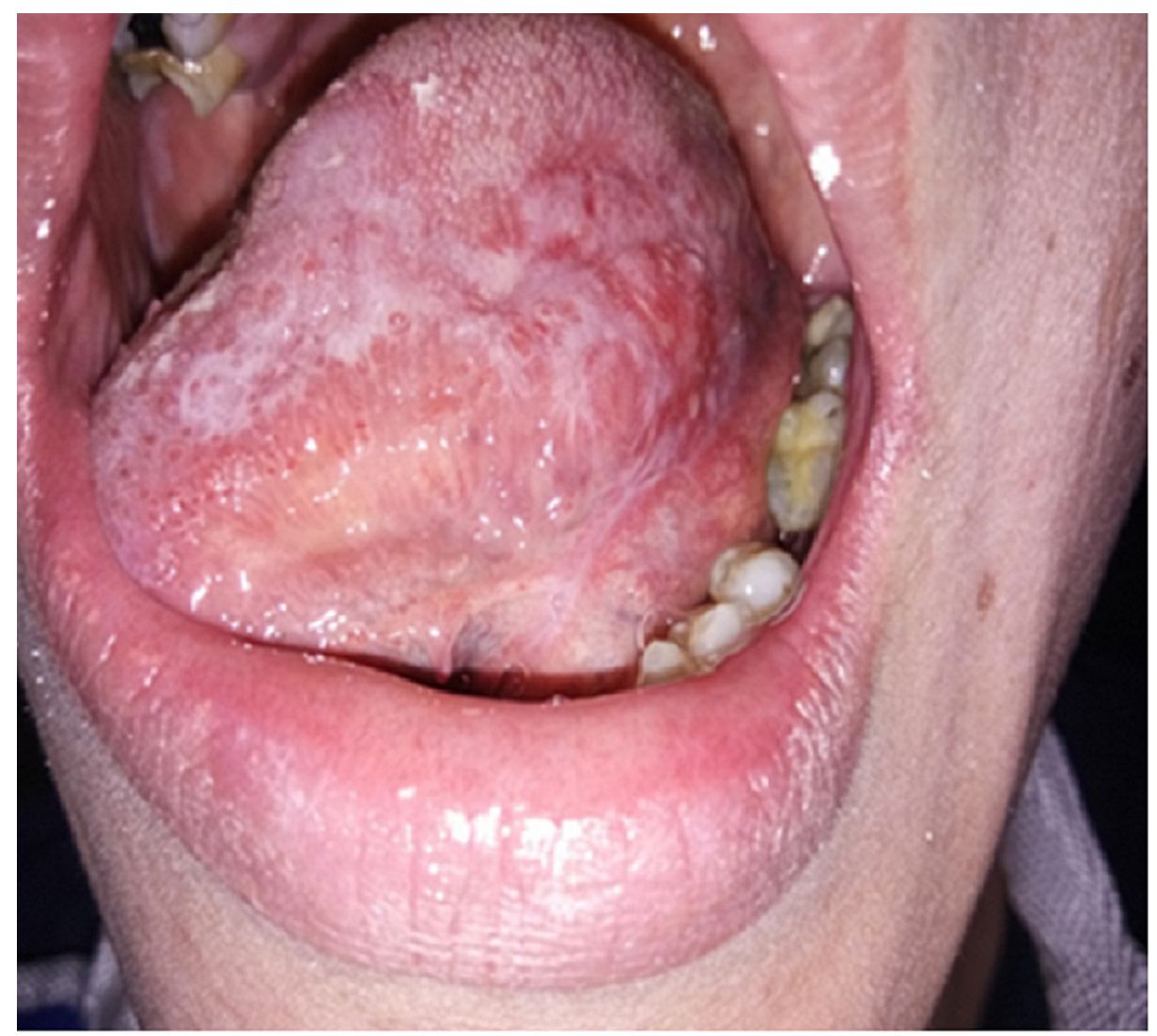

\section{FIGURE 1: Intraoral photograph shows the left lateral border of tongue and dorsum having areas of erythema and white striations on initial presentation.}

An incisional biopsy from the left lateral border of the tongue was performed and sent for routine histopathological examination and direct immunofluorescence. Histopathological findings confirmed OLP. Direct immunofluorescence was negative for fibrinogen, complement protein C3, immunoglobulin (Ig) A, IgG, and IgM, excluding any other immunological mucosal disorder. A skin patch test was carried out using the European standard patch test kit (Chemotechnique diagnostics, Vellinge, Sweden) along with dental material screening series. The patient showed no hypersensitivity to any of the tested substances, including the dental materials.

After establishing the diagnosis of OLP, the patient was started on topical steroid therapy using topical dexamethasone - $1 \mathrm{mg} / \mathrm{g}$ (Dexaltin ${ }^{\circledR}$ oral paste). After initiation of topical steroid therapy, the patient’s symptoms improved, reporting less burning sensation. He was put on follow-up every two to four weeks interval for four months. During these follow-ups, the patient was mainly treated with topical corticosteroids (Dexaltin ${ }^{\circledR}$ ). After four months of follow-up the patient failed to attend the clinic for a period of three months.

During this period the patient had felt extreme lethargy for few days and had sought treatment at a medical facility. On investigation his full blood count revealed severely decreased hemoglobin level which was 6.4 $\mathrm{g} / \mathrm{dl}$ (normal range $13.0-18.0 \mathrm{~g} / \mathrm{dl}$ ); red cell count $2.2 \times 10^{12} / \mathrm{l}$ (normal range $4.5-5.9 \times 10^{12} /$ ); hematocrit 19\% (normal range $41-53 \%$ ). His chest radiograph revealed a mediastinal mass (Figure $2 A, 2 B$ ) which was diagnosed as a thymoma. 


\section{Cureus}
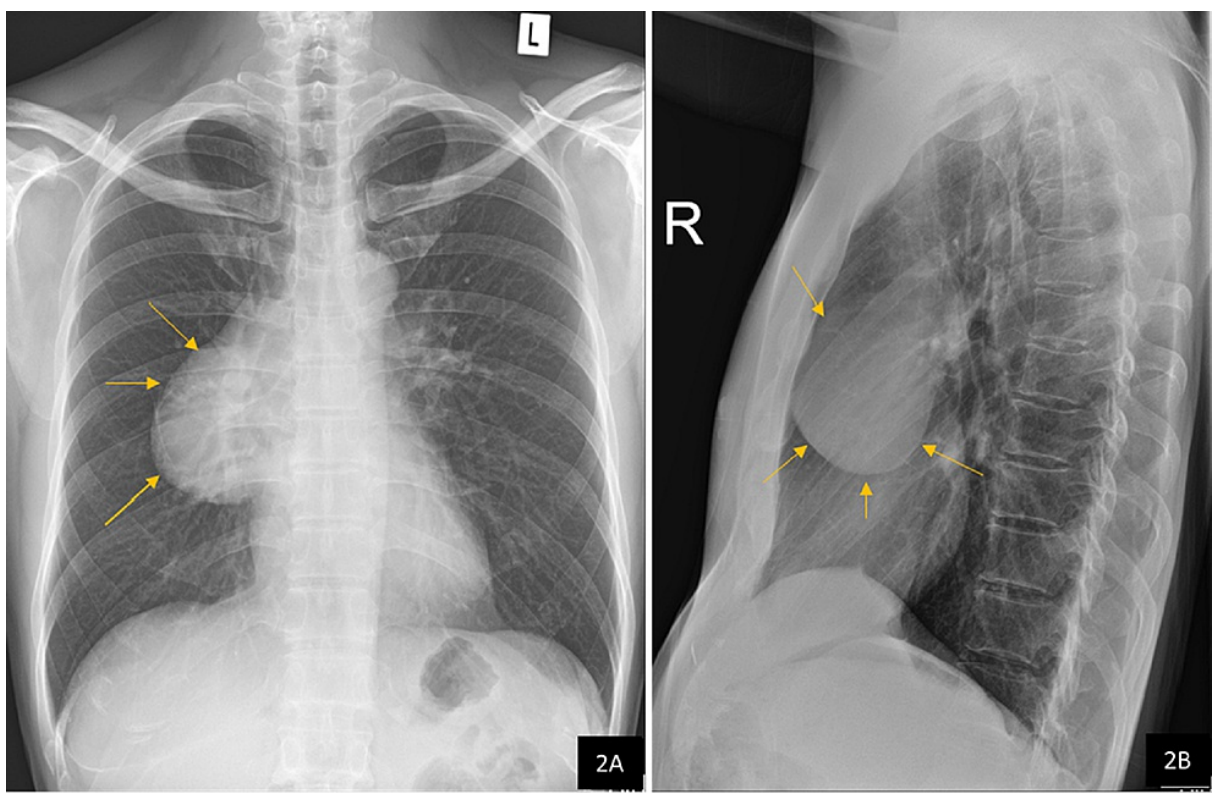

FIGURE 2: Chest radiographs (A) posteroanterior view and (B) lateral view show right paravertebral smooth convex mass overlying the hilum.

His thymoma was treated by surgical excision. The pathology report revealed a mixture of lymphocyte-poor type A and lymphocyte-rich type B thymoma components. The type A component consisted of plump, short, spindle-shaped tumor cells, and type B presented with predominantly mature lymphocytes along with scattered polygonal cells with a vesicular nucleus (Figure 3). The tumor had invaded the capsule and extended into the peri-tumoral adipose tissue. It was diagnosed as type AB thymoma (WHO Classification), minimally invasive.

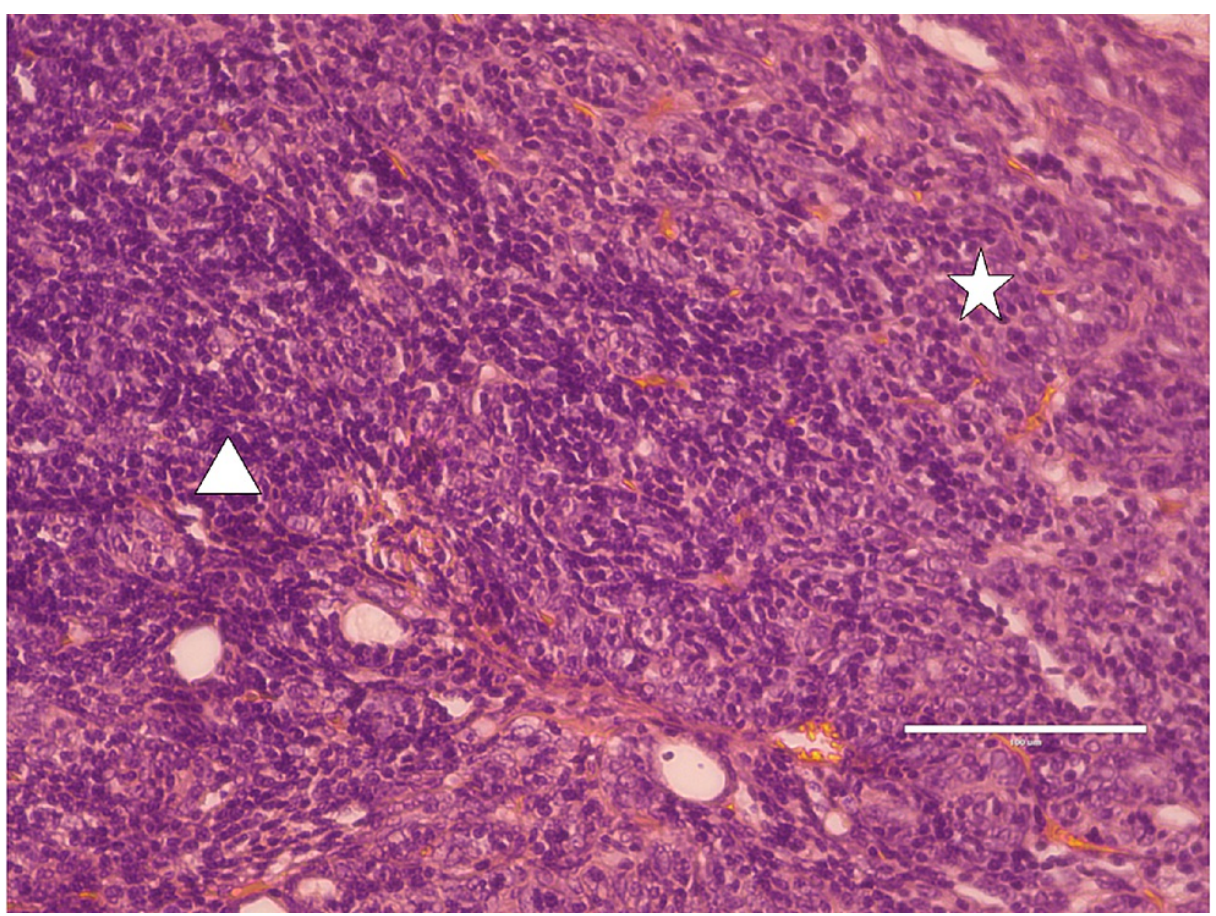

FIGURE 3: Photomicrograph shows a mixture of lymphocyte-poor type A (star) and lymphocyte-rich type B (triangle) components (H\&E, X400).

H\&E - Hematoxylin and Eosin stains 


\section{Cureus}

the hematology reports, was undertaken. A bone marrow trephine biopsy revealed markedly hypocellular marrow, with $5 \%$ cellularity, and the erythroid precursors were markedly reduced. The marrow trephine was interpreted as severely hypocellular marrow, consistent with pure red cell aplasia (PRCA). Thymomaassociated PRCA was established and the patient was treated with systemic cyclosporine $100 \mathrm{mg}$ daily and while on cyclosporine therapy his OLP lesions resolved and became asymptomatic (Figure 4). However, he developed generalized gingival overgrowth which was due to cyclosporine (Figure 5). A diagnosis of druginduced gingival overgrowth secondary to cyclosporine was established and he received non-surgical periodontal treatment for plaque control.

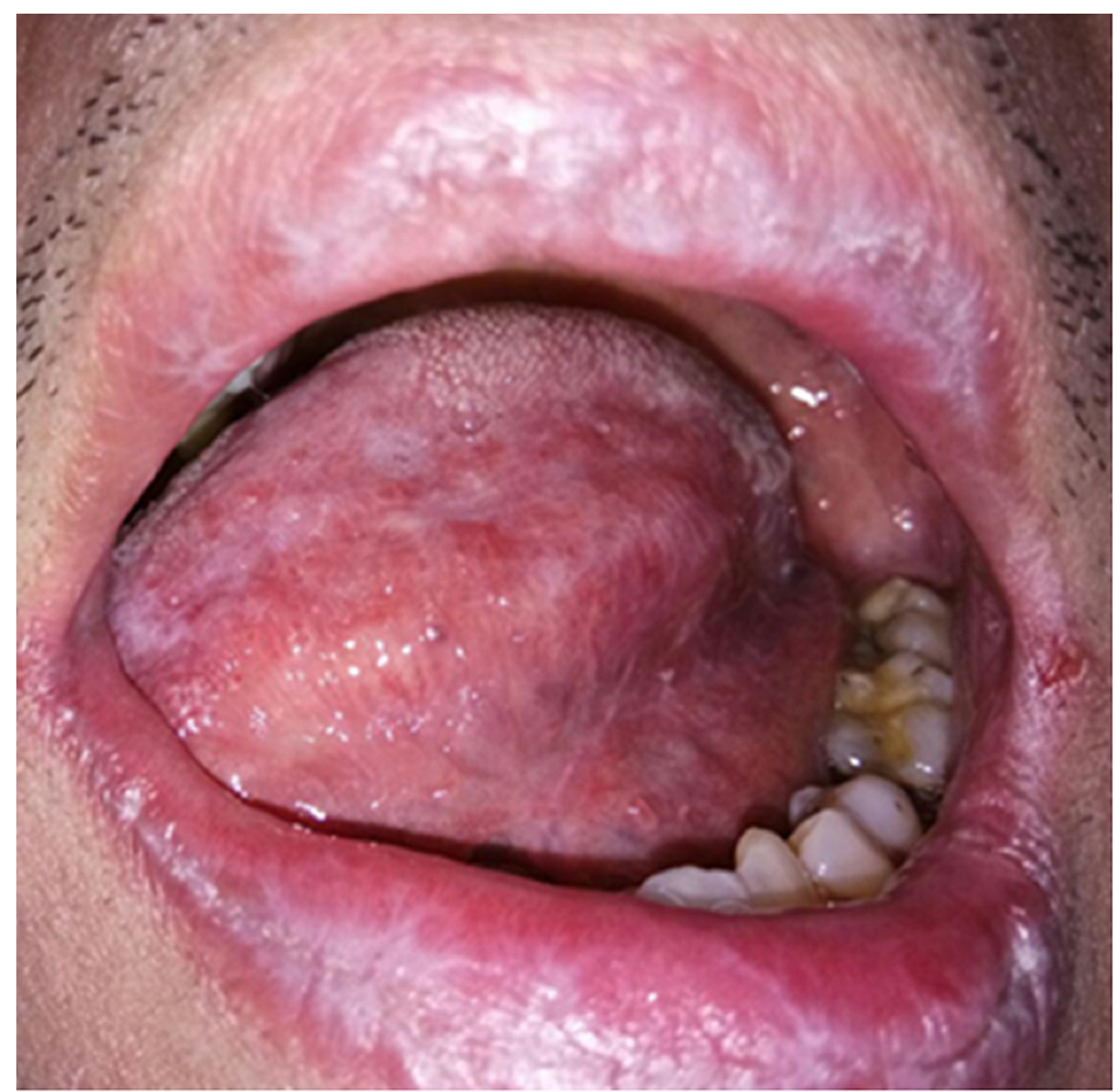

FIGURE 4: Intraoral photograph shows left lateral border of the tongue and dorsum having mild erythema after taking systemic cyclosporine. 


\section{Cureus}

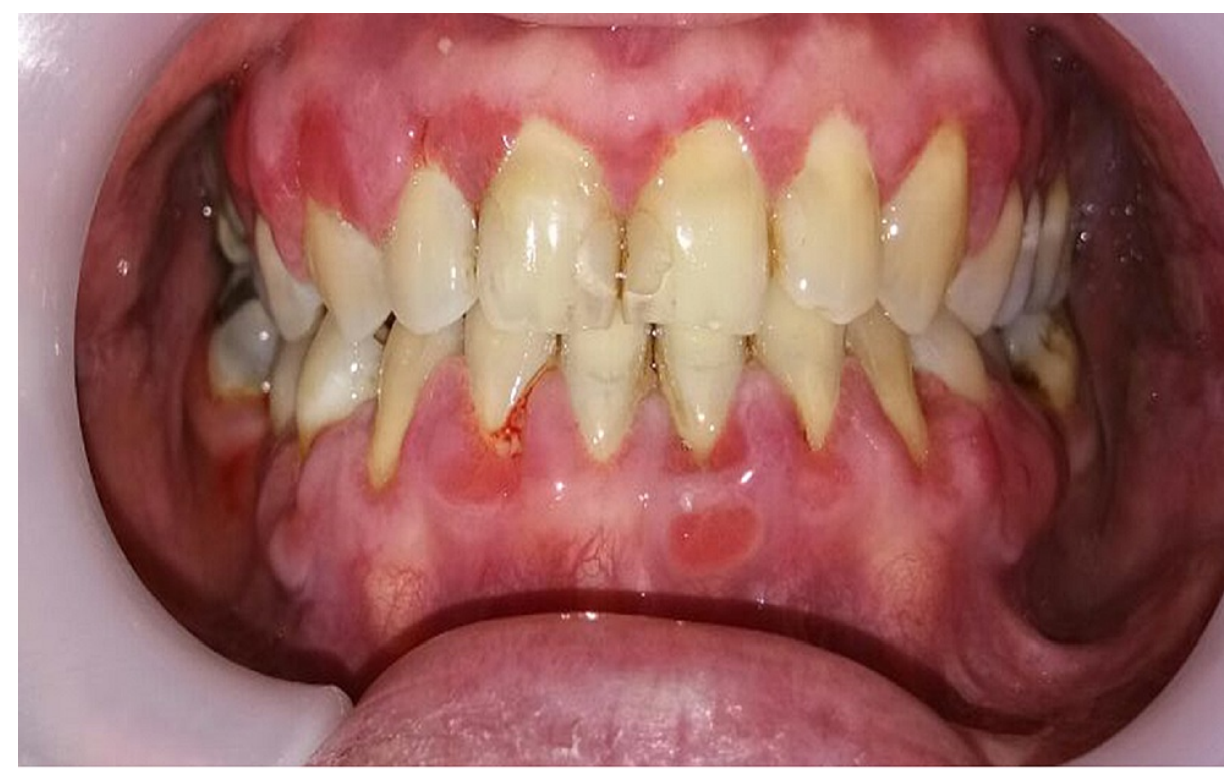

FIGURE 5: Intraoral photograph shows mild generalized gingival growth after taking systemic cyclosporine.

Over three to six months, his cyclosporine dosage was gradually reduced and the gingival overgrowth started to resolve. However, his OLP recurred with white striations, and erythema and ulceration developed on his right and left buccal mucosa, the tongue, and lips (Figure 6). 


\section{Cureus}

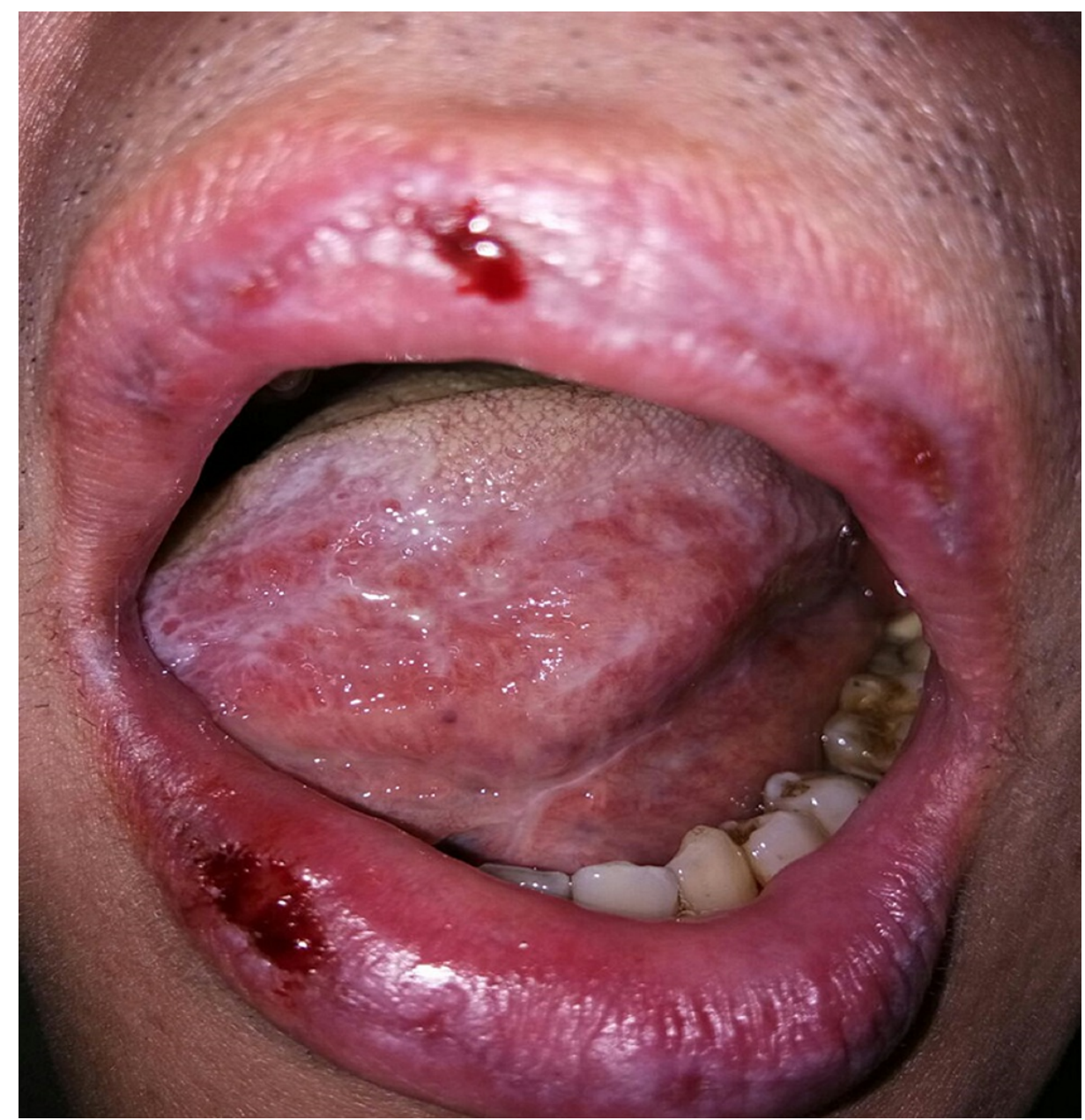

FIGURE 6: Intraoral photograph shows left lateral border of the tongue and dorsum having erythema and ulceration on the upper lip after reducing the dose of systemic cyclosporine.

The patient was further managed with topical steroids and oral hygiene care to control his gingival overgrowth (Figure 7). 


\section{Cureus}

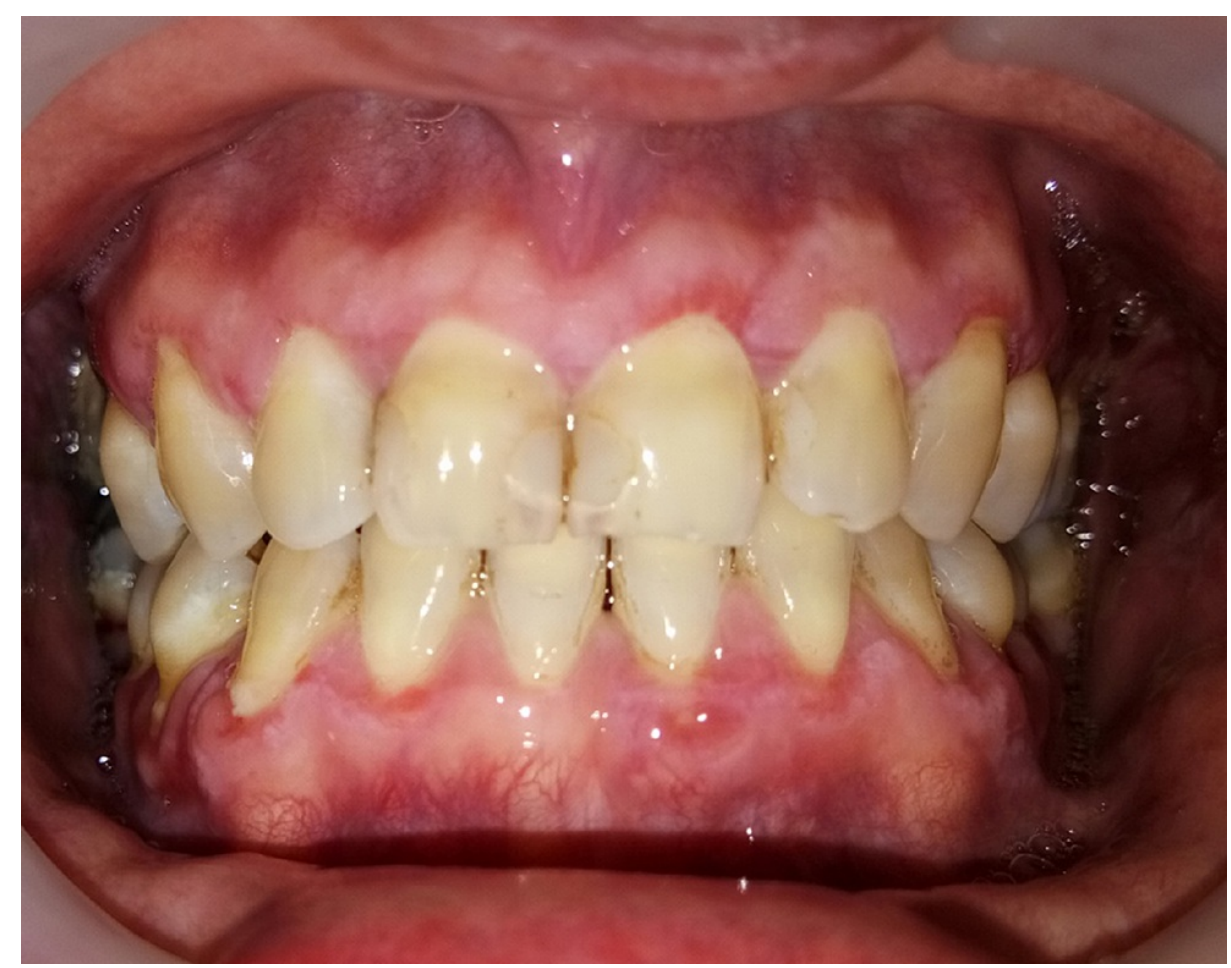

\section{FIGURE 7: Intraoral photograph shows that the generalized gingival overgrowth has resolved following oral hygiene improvement and prophylaxis.}

After stopping the cyclosporine therapy, several flare-ups of his OLP were observed. The patient was managed mainly with topical corticosteroids (Dexaltin ${ }^{\circledR}$ ), dexamethasone mouthwash, and intermittent short-term oral prednisolone $5 \mathrm{mg}$ daily dose given for two weeks. The patient's OLP lesion is overall moderately controlled with a topical corticosteroid (Dexaltin ${ }^{\circledR}$ ). However, the patient responded well to systemic steroids, as the resolution of the lesion is seen after dosages of prednisolone $5 \mathrm{mg}$ for two weeks.

\section{Discussion}

Thymoma is a rare neoplasm of the thymic epithelial cells which commonly occurs in middle-aged individuals [3]. Approximately $30 \%$ of thymomas are asymptomatic, while non-specific symptoms such as cough, dyspnea, and superior vena cava syndrome are seen in another 30-40\% of cases [3]. The etiology of thymoma is unknown, and most cases were detected incidentally during chest imaging for other reasons [4]. Thymomas are commonly associated with autoimmune disorders, the most common being myasthenia gravis [3]. Less frequently, in a small percentage of thymoma patients, hypogammaglobulinemia can develop to form Good Syndrome [5]. Good syndrome was first reported in 1954, it is characterized by adult-onset immunodeficiency, where the patient presents with thymoma and immunodeficiency [6]. The immunodeficiency is characterized by hypogammaglobulinemia and severely reduced or absent B-cells, CD4 lymphopenia, and CD8 lymphocytosis [5]. The association of Good Syndrome with erosive OLP, where patients presented with hypogammaglobulinemia, thymoma, and OLP has been reported [7].

Possible association of thymoma with OLP was first reported in the early 1970s [8]. Lichen planus had been reported in $0.4-1.1 \%$ of patients with thymoma [2]. Most of these patients presented with erosive OLP [2]. Paradoxically, lichen planus in a thymoma patient usually does not regress with thymectomy, which is the primary treatment of thymoma [2, 9]. OLP in patients with thymoma was reported to be resistant to treatment in most reported cases [10]. The pathogenesis of association between OLP and thymoma is unknown [10]. Miyagaki et al. proposed that abnormal regulation of lymphocytes within the thymus may cause OLP [11], and thymoma should be ruled out in patients with intractable lichen planus [10].

Pure red cell aplasia (PRCA) is a syndrome defined by normocytic anemia with severe reticulocytopenia and marked reduction or absence of erythroid precursor from the bone marrow [12]. PRCA can be congenital or acquired. Primary acquired PRCA is an autoimmune disorder in which the erythroid differentiation is interrupted by an immune mechanism, while secondary acquired PRCA is often associated with disorders such as collagen vascular disorders, lymphoproliferative disorders, parvovirus infections, and thymoma [12]. Thymoma is known as the disorder that has the highest association with secondary PRCA, and its discovery can precede the diagnosis of thymoma or can also be discovered after thymectomy [12], as in this 
case. As observed in this case, the management of thymoma-associated PRCA is chiefly by immunosuppressive therapy, where cyclosporine and corticosteroids are the immunosuppressive agents of choice [13].

The main management for thymoma consists of surgical removal or debulking [14], and the most important indicator for prognosis is the completeness of tumor resection [15]. Surgical removal can reduce the possibility of locally invasive growth and metastasis of thymomas; moreover, it usually has a favorable effect on associated disorders such as myasthenia gravis and PRCA [14].

\section{Conclusions}

The purpose of this article is to report an unusual case of OLP which was recalcitrant to treatment. Although initial systemic evaluation did not reveal any associated medical condition, a thymoma was discovered as an occult condition during follow-up. This report provides further evidence that OLP can occur in association with thymoma. The medical-dental interface is important in managing oral mucosal diseases in association with dermatologists, and with specialists in immunology and endocrinology. This case report also highlights the importance of thorough medical history recording in patients presenting with recalcitrant OLP lesions. Based on the current evidence, diagnosis and follow-up of OLP are all the more important due to its potential malignant transformation to oral carcinoma and its association with several systemic diseases, a rare presentation being illustrated in this case.

\section{Additional Information \\ Disclosures}

Human subjects: Consent was obtained or waived by all participants in this study. Conflicts of interest: In compliance with the ICMJE uniform disclosure form, all authors declare the following: Payment/services info: All authors have declared that no financial support was received from any organization for the submitted work. Financial relationships: All authors have declared that they have no financial relationships at present or within the previous three years with any organizations that might have an interest in the submitted work. Other relationships: All authors have declared that there are no other relationships or activities that could appear to have influenced the submitted work.

\section{References}

1. Carrozzo M, Porter S, Mercadante V, Fedele S: Oral lichen planus: a disease or a spectrum of tissue reactions? Types, causes, diagnostic algorhythms, prognosis, management strategies. Periodontol 2000. 2019, 80:105-25. 10.1111/prd.12260

2. Bernard C, Frih H, Pasquet F, et al.: Thymoma associated with autoimmune diseases: 85 cases and literature review. Autoimmun Rev. 2016, 15:82-92. 10.1016/j.autrev.2015.09.005

3. Robbins Basic Pathology E-book. Chapter 12: Disorders of The Thymus. Kumar V, Abbas AK, Aster JC (ed): Elsevier Health Sciences, 2017. 10th Edition:493.

4. Minervini F, Kocher GJ: When to suspect a thymoma: clinical point of view . J Thorac Dis. 2020, 12:7613-18. 10.21037/jtd-2019-thym-05

5. Kelleher P, Misbah SA: What is Good's syndrome? Immunological abnormalities in patients with thymoma . J Clin Pathol. 2003, 56:12-6. 10.1136/jcp.56.1.12

6. GO RA, MA WF: Chest disease in patients with agammaglobulinemia . Dis Chest. 1956, 29:9-35. 10.1378/chest.29.1.9

7. Macdonald JB, Mangold AR, Connolly SM: Good syndrome and lichen planus: case and review. J Eur Acad Dermatol Venereol. 2014, 28:1828-30. 10.1111/jdv.12378

8. Tan RS: Thymoma, acquired hypogammaglobulinaemia, lichen planus, alopecia areata . Proc R Soc Med. 1974, 67:196-8

9. Thymoma and thymic carcinoma treatment (adult) (PDQ $\left.{ }^{\circledR}\right)$-health professional version. (2019). https://www.cancer.gov/types/thymoma/hp/thymoma-treatment-pdq.

10. Motegi S, Uchiyama A, Yamada K, Toki S, Amano H, Ishikawa O: Lichen planus complicated with thymoma: report of three Japanese cases and review of the published work. J Dermatol. 2015, 42:1072-7. 10.1111/13468138.12987

11. Miyagaki T, Sugaya M, Miyamoto A, Nagata M, Ichimura Y, Mitsui H, Sato S: Oral erosive lichen planus associated with thymoma treated with etretinate. Australas I Dermatol. 2013, 54:e25-7. 10.1111/j.14400960.2011.00818.x

12. Means RT Jr: Pure red cell aplasia. Blood. 2016, 128:2504-9. 10.1182/blood-2016-05-717140

13. Sawada K, Fujishima N, Hirokawa M: Acquired pure red cell aplasia: updated review of treatment . Br J Haematol. 2008, 142:505-14. 10.1111/j.1365-2141.2008.07216.x

14. Kelesidis T, Yang O: Good's syndrome remains a mystery after 55 years: a systematic review of the scientific evidence. Clin Immunol. 2010, 135:347-63. 10.1016/j.clim.2010.01.006

15. Johnson SB, Eng TY, Giaccone G, Thomas CR Jr: Thymoma: update for the new millennium. Oncologist. 2001, 6:239-46. 10.1634/theoncologist.6-3-239 